Archives de sciences sociales des religions

149 | janvier-mars 2010

Varia

\title{
La tombe de Victor Noir au cimetière du Père- Lachaise
}

\section{Marina Emelyanova-Griva}

\section{OpenEdition}

12 Journals

Édition électronique

URL : http://journals.openedition.org/assr/21870

DOI : 10.4000/assr.21870

ISSN : $1777-5825$

Éditeur

Éditions de l'EHESS

Édition imprimée

Date de publication : 31 mars 2010

Pagination : 89-108

ISBN : 978-2-7132-2253-5

ISSN : 0335-5985

Référence électronique

Marina Emelyanova-Griva, "La tombe de Victor Noir au cimetière du Père-Lachaise ", Archives de sciences sociales des religions [En ligne], 149 | janvier-mars 2010, mis en ligne le 01 mars 2013, consulté le 21 décembre 2020. URL : http://journals.openedition.org/assr/21870 ; DOI : https:// doi.org/10.4000/assr.21870 


\section{Marina Emelyanova-Griva}

\section{La tombe de Victor Noir au cimetière du Père-Lachaise}

Le cimetière du Père-Lachaise est le plus connu des vingt cimetières parisiens. Ouvert en 1804, il totalise à ce jour environ soixante-dix mille concessions et accueille chaque année plus de deux millions de visiteurs venus du monde entier. Au-delà de sa fonction première, la célèbre nécropole est devenue un véritable musée en plein air ainsi qu'un jardin panthéon : des centaines de personnalités des arts et des lettres, des sciences et de la politique y reposent. Les visiteurs se promènent dans le cimetière mais beaucoup y viennent spécialement pour voir les tombes ou les monuments qui les intéressent. Certaines tombes font même l'objet d'un véritable culte. Nombreux, par exemple, sont les amoureux qui viennent se recueillir autour du cénotaphe d'Héloïse et Abélard. Plus nombreux encore sont ceux qui se rassemblent autour du mausolée-dolmen d'Allan Kardec dont on dit que le fait d'imposer la main sur son buste permet la réalisation des vœux. La tombe de Jim Morrison, ancien chanteur du groupe des Doors, attire un grand nombre de représentants de la rock génération: musique et graffiti sont, en général, au programme du culte qui lui est constamment rendu. La sépulture de Victor Noir, objet de cet article, est l'une des plus fréquentées. Ce journaliste du XIX ${ }^{\mathrm{e}}$ siècle, sous la forme d'un beau gisant de bronze, œuvre de Jules Dalou ${ }^{1}$, est devenu une sorte de symbole de la fécondité. Sa tombe présente la figure du jeune homme allongé, les mains étendues le long du corps, son chapeau à ses pieds. On remarque vite que certaines parties du gisant brillent, objets de nombreux attouchements: la légende veut qu'en frottant le gisant, surtout à l'endroit de son sexe, on retrouve la fécondité ou la virilité.

1. Jules Dalou (1838-1902), sculpteur français, représentant du naturalisme en sculpture, élève de Carpeaux, auteur du Triomphe de la République, place de la Nation à Paris. Il participa à la Commune et dut se réfugier en Angleterre jusqu'en 1879. 


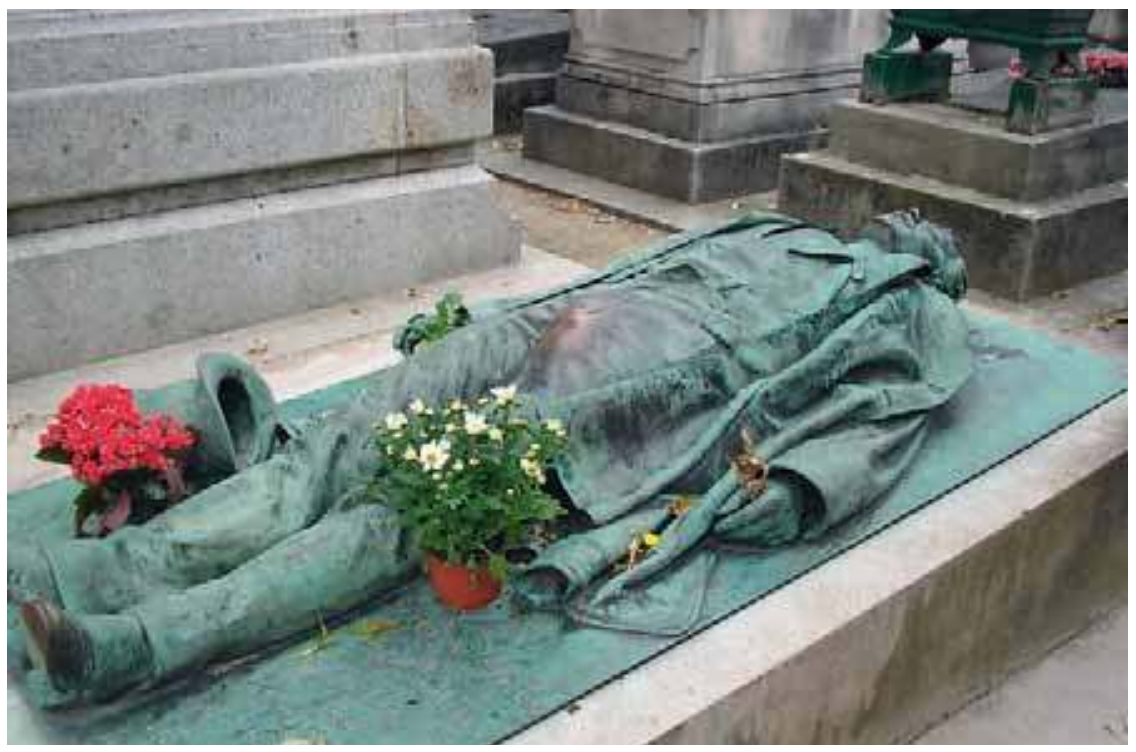

Le gisant de Victor Noir (photo de l'auteur, octobre 2005)
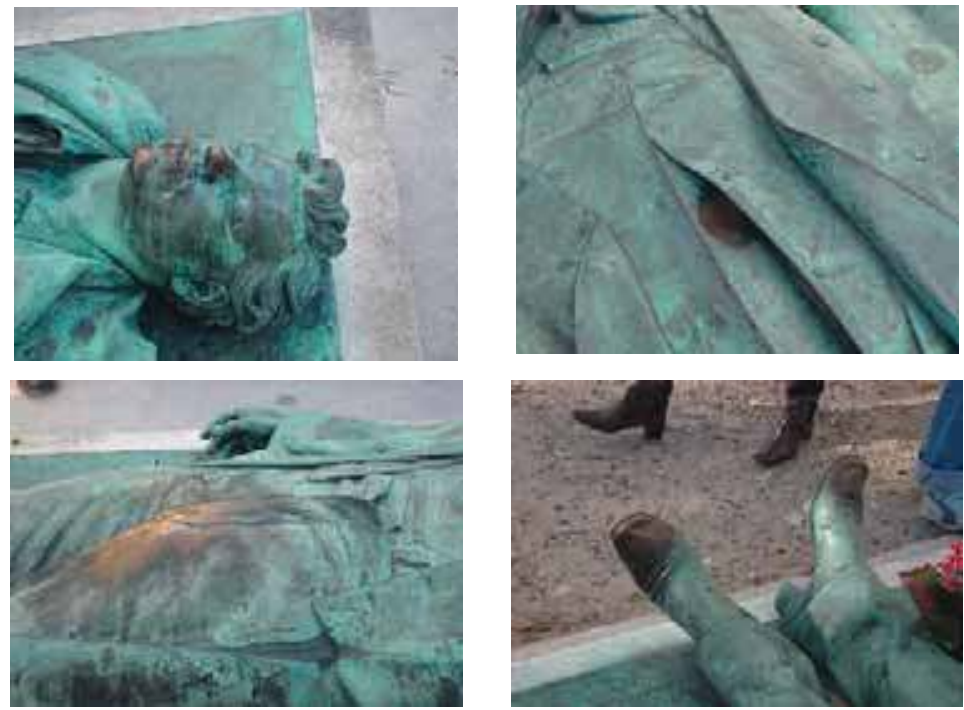

Les parties lustrées de la statue de Victor Noir (photos de l'auteur, octobre 2005)

«Le monument figure dans un grande nombre de genres du folklore urbain - dans des anecdotes, dans des récits mythologiques et quotidiens, dans des rites, qui accompagnent la "vie" de la plupart des monuments de leur installation jusqu'à la destruction. Le thème du monument est actuel pour la culture moderne dans son ensemble. Un monument dans une ville a plusieurs fonctions, avant tout 
une fonction de transmission de la mémoire culturelle et historique. Mais en réalité cette fonction n'est pas unique et même pas principale pour un monument » (Lourie, 2003 : 420). Dans cet article, je voudrais montrer quelles transformations fonctionnelles a connu le gisant de Victor Noir depuis son inauguration jusqu'à nos jours.

De son vrai nom Yvan Salmon, Victor Noir a commencé sa vie active comme apprenti horloger chez son père, puis comme fleuriste, avant de devenir journaliste. Il a travaillé au Journal de Paris, au Corsaire, au Figaro, au Rappel avant d'entrer à La Marseillaise, périodique lancé par Henri Rochefort ${ }^{2}$. Le 10 janvier 1870, il est tué par le prince Pierre Bonaparte ${ }^{3}$. Au cours d'une polémique de presse entre Pierre Bonaparte et Pascal Grousset, également journaliste de La Marseillaise, ce dernier s'est prétendu offensé et a envoyé à Auteuil, au domicile du prince, deux témoins - Victor Noir et Ulric de Fonvielle - pour demander réparation par les armes. On ne sait pas ce qui s'est passé au domicile du prince : seulement que dans un mouvement de colère ou de défense, Pierre Bonaparte tue Victor Noir. Cet événement est incompréhensible si on ne le replace pas dans l'atmosphère de l'époque : «La brusque nouvelle du meurtre de Victor Noir jette la consternation chez l'Empereur et jette le désarroi dans les milieux gouvernementaux. Depuis une année, Napoléon III se nourrit de l'espérance d'apaiser quelques revendications et de regagner, au centre et sur la lisière de la gauche, quelques partisans, à l'aide de concessions qui lui paraissent opportunes. Les lois du 11 mai 1868 sur la presse et du 6 juin 1868 sur les réunions ont marqué un premier effort dans ce sens... L'Empereur annonce volontiers que, l'ordre maintenant assuré, l'heure de la liberté a sonné désormais, que des réformes constitutionnelles seront réalisées et que, selon la formule fameuse, l'édifice va recevoir son couronnement. À l'Empire autoritaire doit succéder l'Empire libéral. Et déjà cette évolution vers le libéralisme lui vaut des adhésions... N'est-ce point comme une fatalité qu'à cette heure-là le geste malencontreux d'Auteuil vienne fournir aux républicains qui n'oublient pas, aux “irréconciliables” l'occasion d'évoquer les violences et les répressions sanglantes qui ont marqué les débuts du régime... » (Zévaès, 1929 : 36-37). Cet événement n'était donc pas un simple fait divers, puisque le meurtrier était membre de la famille impériale et que la victime était journaliste d'opposition. De fait divers douloureux, l'épisode devient un événement politique. À la veille de la guerre de 1870, le prince Pierre Bonaparte est

2. Henri Rochefort (1831-1913), journaliste et homme politique français. Il aborda le journalisme politique dans Le Nain jaune, Le Soleil, Le Figaro. En 1868 il fonda La Lanterne, pamphlet virulent contre l'Empire. Député de Belleville, en 1869, il rédigea La Marseillaise et fut emprisonné lors du procès de l'affaire Victor Noir puis libéré par la révolution du 4 septembre. Sans appartenir à la Commune, il en approuva l'action et fut emprisonné, puis déporté. Il rentra en France lors de l'amnistie de 1880.

3. Pierre Bonaparte (1815-1881), sixième enfant du second mariage de Lucien. Il combattit en Romagne en 1831, puis en Colombie et en Égypte. Député d'extrême-gauche aux Assemblées de 1848 , il critiqua le 2 décembre et se retira de la vie publique. 
acquitté, au grand scandale de l'opinion. Entre temps, Victor Noir a été enterré à Neuilly où les obsèques du journaliste ont donné lieu à une grande manifestation républicaine et une émotion populaire très vive qui font de lui le symbole de la répression de l'Empire face à la lutte pour la liberté. Victor Noir, journaliste peu connu auparavant, devient un martyr républicain, un héros par son innocence, sa jeunesse et sa mort tragique.

«Dans les années 1880, la Troisième République s’étant définitivement imposée, comme régime politique de la France, le gouvernement décida de rendre un hommage particulier à ce jeune républicain considéré comme une victime innocente du Second Empire. Une souscription publique fut lancée afin de permettre l'édification au cimetière du Père-Lachaise, sur un terrain accordé gratuitement par la Ville de Paris, d'un monument conçu et réalisé par le grand sculpteur Jules Dalou (1838-1902) » ${ }^{4}$.

On peut dès lors parler d'un culte de Victor Noir, apparu juste après sa mort. Mais c'est un culte politique, manifesté dans l'hommage rendu à cet «enfant du peuple " tué par un prince, dans la prise de conscience d'un devoir de reconnaissance publique envers le défunt qui l'a mérité par la brutalité de sa mort et l'héroïsme qu'on lui attribue. "Le $19^{\mathrm{e}}$ siècle a eu un véritable culte du "grand homme". Le développement des cimetières est une conséquence de ce culte. Le succès des cimetières dont la création, en France, est décidée en 1804, repose en effet sur la possibilité qu'ils donnent de prolonger la mémoire, dans une société pour qui l'oubli est la véritable mort " (Le culte des grands hommes..., 1990 : 212) ${ }^{5}$. «Le monument, parfois financé grâce à la souscription nationale, portera témoignage de l'action bienfaitrice du défunt. Les tombes des martyrs ou des héros feront l'objet d'un culte collectif »(Charneau, 1997 : 10-11). Le culte de Victor Noir est de cette qualité. Tous ceux qui nourrissent une sympathie pour la Commune et les Fédérés considèrent Victor Noir comme un martyr, comme une victime de la politique impériale. Son assassinat étant considéré comme un des éléments qui ont conduit au renversement du Second Empire et à la proclamation de la République, nombre de visiteurs du Père-Lachaise qui viennent se recueillir devant le Mur des Fédérés ou devant les tombes des membres de la Commune incorporent Victor Noir dans leur itinéraire. Il faut prendre avec réserve l'idée que la mort de Victor Noir a provoqué la chute de l'Empire bien qu'elle fût sûrement un des facteurs qui ont érodé la légitimité de l'empereur ; c'est la guerre de 1870 qui l'a fait chuter. Cependant, le fait que l'on confère autant d'importance politique au meurtre de Victor Noir est significatif.

4. C. Charlet, Étude sur le tombeau de Victor Noir au cimetière du Père-Lachaise : Légendes et réalité, travail non publié, consulté grâce à l'obligeance de l'auteur.

5. En fait, la loi de prairial an XII a accéléré la translation des cimetières, prévue par l'ordonnance royale de 1776. Sur l'évolution du cimetière en France au XIX siècle et, plus particulièrement, sur le cimetière du Père-Lachaise, voir Lassère, 1997 ; Bertrand, Vovelle, 1983 ; Tartakovsky, 1999. 
Le culte politique de Victor Noir a idéalisé ce journaliste et l'histoire de sa vie et sa mort a poétisé ses dernières minutes. De surcroît, le fait qu'il a été tué deux jours avant son mariage ajoute une touche romantique et sentimentale au récit. Le culte politique de Victor Noir a ainsi laissé le gisant, une œuvre d'une puissance dramatique intense et devenue au fil du temps l'objet de pratiques à caractère sexuel.

Les recherches effectuées pour comprendre quand et comment le culte autour de la tombe de Victor Noir a assumé des connotations sexuelles n'ont pas donné de réponse concluante mais ont permis de trouver des pistes et de distinguer quelques versions. Tout d'abord il faut dire que, malgré un point de vue répandu parmi nos informateurs, le sculpteur Jules Dalou, se réclamant du réalisme, avait voulu représenter le gisant de Victor Noir d'une manière saisissante, au plus près de la "réalité », et n'avait nullement l'intention de mettre en évidence le sexe du journaliste. Ce sont les nombreux frottements qui le font briller et le rendent très visible.

Le monument de Dalou s'inscrit dans le renouveau de l'art du gisant dans la sculpture française du XIX siècle et les premiers commentateurs du gisant insistent sur sa signification à la fois réaliste et pathétique. "Motif de prédilection de la sculpture funéraire depuis le $\mathrm{XI}^{\mathrm{e}}$ siècle, les gisants se multiplièrent entre 1490 et 1530, au moment où les représentations de la mort physique furent aussi les plus nombreuses. Dans la deuxième moitié $\mathrm{du} \mathrm{XVI} \mathrm{e}^{\mathrm{e}}$ siècle ils diminuèrent et disparurent... Trois siècles plus tard, ils retrouvèrent une place de choix dans les cimetières. Leur réapparition avait été préparée par la représentation du défunt sur son lit de mort, et les premiers gisants sont à mettre en rapport avec ce genre de scènes... L'esprit romantique et le renouveau néo-gothique contribuèrent pour une large part à la résurgence du thème des gisants " (Le NormandRomain, 1995 : 183-184). Dans les cimetières, les gisants n'apparaissent guère avant le milieu du XIX ${ }^{\mathrm{e}}$ siècle (le gisant de Cavaignac par Rude au cimetière de Montmartre étant un des premiers) et se multiplient dans la seconde moitié du siècle. "Les uns insistent sur la mort physique, les autres, au contraire, la nient, prétendant qu'il ne s'agit que de sommeil, ou même simplement de repos, mais, dans les deux cas, le lien avec la Renaissance est évident » (ibid. : 204). "Quoique allongées, certaines statues funéraires ne peuvent pas être assimilées aux gisants traditionnels : les personnages qu'elles présentent sont mourants ou déjà morts, car ce sont les circonstances violentes dans lesquelles ils ont péri qui les caractérisent le mieux. La sculpture funéraire rappelle donc celles-ci, et à partir de 1840, sous l'influence des courants romantiques et réalistes, insiste tout particulièrement sur la brutalité et l'injustice de leur mort. Les œuvres réalisées alors par des artistes qui partagent ces sentiments et mettent leur point d'honneur à les faire partager à leur tour, comptent pour la plupart parmi les belles réussites des cimetières " (ibid. : 227). C'est le cas du gisant de Victor Noir. Après la chute 
du Second Empire, le réalisme l'a emporté sur le romantisme et c'est à cette époque que Dalou crée ce gisant en prétendant montrer la vérité historique sèche et méticuleuse. Mais le réalisme n’implique pas forcément une mise en évidence de la sexualité. Cependant, le gisant a fini par devenir un objet de culte sexuel, ce qui laisse à penser que, même si le sculpteur ne le voulait pas, son œuvre a inspiré des idées équivoques. Ainsi, il est important de comprendre quand et sous quelles formes ces connotations sexuelles apparaissent dans la perception du gisant, et quand et comment elles suggèrent la naissance d'un culte sexuel.

On trouve la première trace de cette transformation sur le dessin du peintre allemand Christian Schad, daté de 1929, qui présente une femme en train de chevaucher le gisant. Les descriptions concernant ce dessin ne donnent pas d'explications concrètes et n'évoquent même pas le nom du journaliste. C'est Gérard Laplantine qui a reconnu sur le dessin la tombe de Victor Noir et qui l'évoque dans Inscriptions lapidaires et traces de passages. Considérant le dessin de Schad comme la preuve qu'une perception sexuelle existait déjà en 1929, l'auteur écrit : "Cette date nous instruit sur la rapidité de la formation d'une pratique votive: soit dans ce cas que le bronze du gisant fut dès le début un sujet de plaisanterie remarqué et développé par les curieux et artistes du moment, soit que la dévotion ait précédé et provoqué leur attention » (G. Laplantine, 1993 : 145).

En s'appuyant sur les recherches effectuées, on peut reformuler cette hypothèse en précisant sa première version : étant donné que ce dessin est l'unique source présentant la pratique sexuelle (ou plutôt sa caricature) liée au gisant avant les années soixante, c'est ce peintre qui illustre ou même impulse «la plaisanterie ", la pratique imaginaire relative à ce monument dans le milieu artistique. On trouve des preuves indirectes de cette hypothèse dans la personnalité même de ce peintre avant-gardiste dont les thèmes majeurs étaient les travestissements et les bizarreries sexuelles. Il avait une passion pour «l'étude des pulsions et des déviances sexuelles » (Lloyd, 2002 : 23) et considérait Paris comme une ville idéale, le "symbole de la bohème artistique et de la modernité... lieu où tout peut arriver " (ibid. : 20). De surcroît, comme le relate un de ses commentateurs, «Schad a parfois utilisé le fusain pour des études préparatoires de grand format en rapport avec ses portraits, tandis que les petits dessins à la plume de la période 1927-1930 présentent des compositions imaginaires souvent liées à des commandes d'illustration » (ibid.). Également significatif : c'est qu'à partir de la parution de ce dessin et jusqu'aux années soixante, on n'a trouvé aucune autre trace de changement de sens du culte. C'est du milieu des années soixante, quand l'information sur les pratiques sexuelles autour de la tombe de Victor Noir pénètre dans la littérature populaire, qu'il faut dater la véritable naissance du culte sexuel et le début de sa popularisation. Il est difficile de mesurer le rôle du dessin de Schad dans ce processus ; mais on peut supposer, bien que l'hypothèse demeure 
hasardeuse, que cela est lié aux années soixante et que les travaux de Schad de la période d'entre-deux-guerres sont redécouverts par des critiques et des marchands.

Une autre version de la mutation du culte politique en culte sexuel, de l'étonnante transformation de l'image du héros national, martyr de la République, en sex-symbol du cimetière est donnée par Christian Charlet. Selon lui, " de 1891 à 1942, le gisant de Victor Noir, entouré et protégé du public par une belle chaîne de bronze également œuvre de Dalou, fut totalement respecté par les visiteurs ${ }^{6}$. Pendant l'occupation de la France par l'Allemagne (1940-1944), les autorités d'Occupation firent fondre à Paris un grand nombre de statues et autres ouvrages de bronze pour la fabrication de canons. M. Charlet affirme que "la chaîne du tombeau de Victor Noir subit le même sort et le gisant se trouva alors privé de sa protection, à la merci des visiteurs ».

Il a également raconté l'événement qui, à son avis, est à l'origine du culte : "Un groupe d'étudiants, confondant cimetière et lieu de rigolade, inventa au début des années soixante un canular reposant sur la prétendue virilité de Victor Noir ainsi que de Blanqui dont le gisant n'avait jamais été protégé. Suffisamment aisés pour pouvoir corrompre un fonctionnaire, ils achetèrent le concours d'un gardien du Père-Lachaise pour que celui-ci frotte consciencieusement et régulièrement les emplacements présumés des organes sexuels de Blanqui et de Victor Noir, de manière à les mettre en évidence, bien lustrés, sur les gisants alors couverts de vert-de-gris (oxydation du bronze), faute d'entretien depuis des années ${ }^{7}$. Les conséquences du canular étaient que "peu de temps après, un journaliste intéressé exploita de façon lucrative ce canular d'étudiants à base de corruption et inventa purement et simplement un faux culte de la fécondité et de la virilité dans lequel le fait pour des visiteurs de se frotter les organes sexuels sur ceux des gisants développerait soi-disant la virilité des hommes et surtout la fécondité des femmes, oubliant que jusqu'alors les profanations de tombeaux - c'en est une - étaient réputées porter malheur aux profanateurs ${ }^{8}$. Une autre version lie directement l'apparition du culte en sens sexuel à l'activité des journalistes qui auraient inventé ce culte et l'auraient popularisé dans la presse. Faute de pouvoir étayer ces hypothèses, on peut seulement les admettre comme des versions assez vraisemblables.

Les premiers renseignements trouvés dans la littérature populaire sur le culte de la fécondité autour de la tombe de Victor Noir datent de 1966. À la fin des années soixante et au début des années soixante-dix, le gisant de Victor Noir était probablement vénéré par un nombre limité de personnes. Mais à partir de la fin des années soixante-dix, et de façon continue, de nombreux livres sur le

6. Christian Charlet, note 5, p. 2.

7. Ibid.

8. Ibid. 
cimetière du Père-Lachaise parlent de ce culte, décrivent les rites qui l'accompagnent, ignorant souvent l'histoire réelle de la mort du journaliste, ce qui a agrandi le cercle des visiteurs et fait de la tombe de Victor Noir l'une des plus fréquentées du cimetière. Les touristes viennent la voir, la photographier, se prendre en photo avec un geste caractéristique (la main sur le sexe du journaliste), la toucher en formulant un vœu. Les guides officiels ou dilettantes (il y en a beaucoup au Père-Lachaise) jouent un rôle de vecteurs et de transmetteurs de l'information, et assument facilement un rôle de créateurs de représentations et de rites.

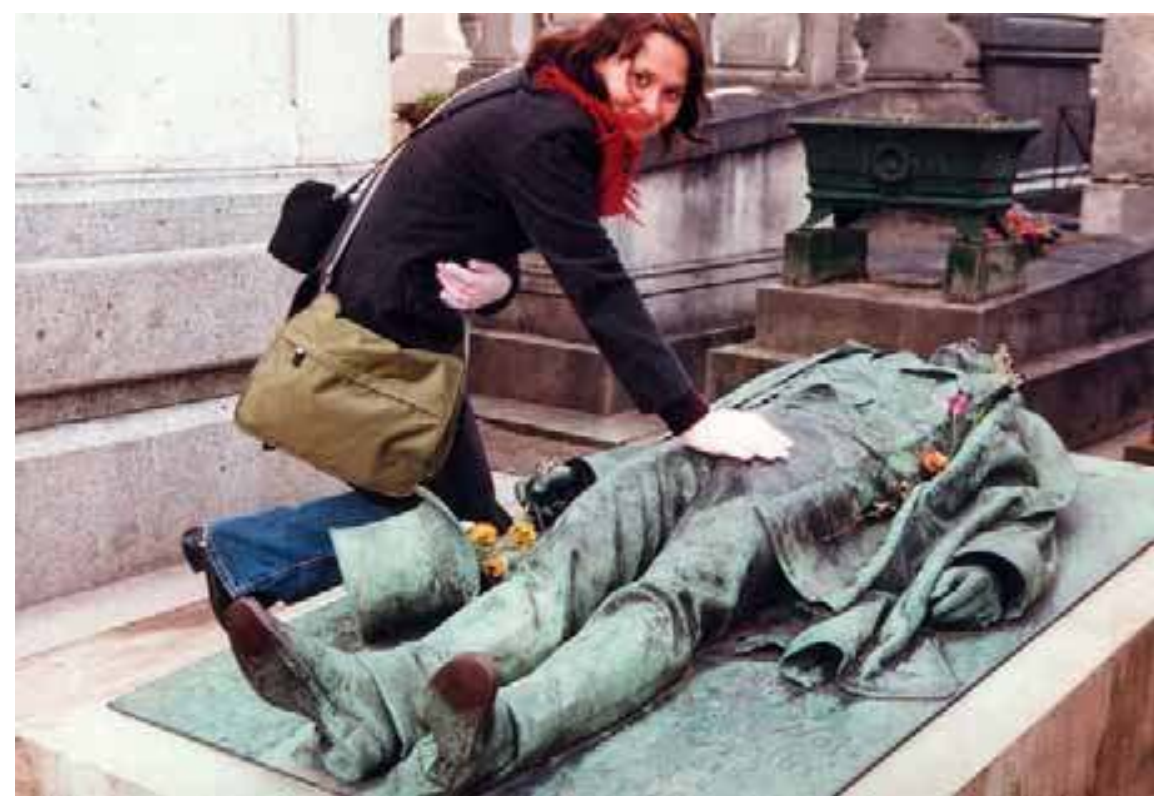

Le geste typique pour les photos-souvenirs avec le gisant de Victor Noir (photo de Michael Majzulse, mai 2005)

Notons que les guides se séparent clairement. Il y a ceux qui préfèrent ne pas parler du culte ou en parler brièvement et avec précaution. Les autres, soit ne parlent que du culte en faisant la démonstration des rites et laissent un peu de temps pour que les membres du groupe profitent de l'occasion et vérifient les pouvoirs magiques du gisant, soit évoquent à peine la mort du journaliste en commettant beaucoup d'erreurs. Les visiteurs semblent préférer les seconds... Mais la tombe n'est pas seulement vénérée par des touristes : les Français viennent en tant que guides pour montrer la tombe célèbre à leurs amis, pour passer du temps et se divertir en voyant la statue si souvent frottée ou pour pratiquer de véritables rites s'ils croient aux pouvoirs magiques du gisant. 
L'étude de la situation actuelle de la tombe de Victor Noir se base sur un travail de terrain effectué par l'auteur et son mari, Griva Viacheslav, qui ont observé ce qui se passait autour de la tombe pendant vingt jours. Les observations se sont déroulées essentiellement de mai à juillet 2006. On a pratiqué la technique de l'observation participante et passive, de l'observation directe et indirecte et celle des entretiens. Selon François Laplantine, "l'ethnographie est d'abord une activité visuelle » (2002: 7) qui consiste non seulement à voir, mais "à faire voir ". De là découle le rôle de la description ethnographique. Sans toujours pouvoir reproduire toutes les paroles d'un informateur - d'autant plus que, souvent, il n'apprécie pas de voir noter ce qu'il dit -, on a tenté d'effectuer des $"$ descriptions denses ${ }^{9}$ : descriptions les plus précises possible de l'attitude, de la gestuelle, de la mimique afin de dépasser les seules représentations et d'inclure les normes et les stratégies de conduite. Comme le remarque A. Van Gennep : "L'observateur se trouve ainsi forcé de noter en même temps plusieurs faits de détail groupés autour d'un noyau central ; mais chacun de ces détails peut dans d'autres conditions servir aussi de noyau » (1982: 59).

Il faut remarquer que la spécificité des pratiques opérées sur la tombe de Victor Noir crée beaucoup de difficultés d'observation et d'interrogation. Le plus souvent, les gens qui croient aux pouvoirs magiques du gisant préfèrent passer inaperçus et n'acceptent pas d'être observés ou interrogés. Cependant, les vrais "pratiquants ", fréquentant la tombe régulièrement, expliquent leurs représentations et montrent les rites assez volontiers. Dans tous les cas, l'observation demeure une source très riche, surtout si on utilise la méthode de E. Gofman qui enseigne l'observation des gestes, des modes d'expression les plus insignifiants mais cependant jamais neutres: "Les gestes que parfois nous nommons vides sont peut-être, en fait, les plus pleins de tous » (1974: 81). En ce qui concerne le questionnaire, il évoluait en fonction de la situation et se modifiait continuellement. Par exemple, la première question variait entre «Pourquoi le gisant est-il abîmé comme ça ? » (pour ceux qui critiquent l'existence du culte) et "Qu'est-ce qu'il faut faire pour se marier ? " (pour ceux qui pratiquent des rites eux-mêmes). Après ces premières questions, on préférait laisser parler les informateurs avant de leur demander, finalement, leur source d'information et leur point de vue concernant la date de la naissance du culte.

Ce travail de terrain a permis de distinguer différents types de visiteurs; de mettre en évidence le rôle des guides; de relever le mécanisme de transmission de l'information sur le culte; de faire la liste des propriétés magiques attribuées au gisant de Victor Noir; d'analyser les pratiques rituelles autour de la tombe en conformité avec les représentations liées au gisant, etc. Ainsi, parmi la grande quantité d'informations recueillies, on a pu observer certaines régularités permettant une analyse plus fine du phénomène.

9. Le terme de Clifford Geertz qu'il a emprunté au philosophe Gilbert Ryle. 
Il n'y a aucune difficulté à trouver cette tombe : elle est indiquée sur le plan du cimetière et le premier passant venu vous montre le chemin. Les habitués $\mathrm{du}$ cimetière, les "pèrelachaisiens " comme les appellent les gardiens - des personnes âgées, des mères avec leurs bébés ou d'autres qui y viennent régulièrement prendre l'air comme dans un parc -, n'entrent pas, d'habitude, dans l'espace examiné autour de la tombe de Victor Noir mais passent devant la tombe. Cependant les phrases qu'ils échangent en passant à côté sont souvent très intéressantes : "Ah, c'est celui qui est frotté ! ", dit une femme en parlant avec sa fille ${ }^{10}$; deux Françaises de dix-huit ans, en passant à côté de la tombe : «-Ah, c'est ça... on frotte dessus ! - Non, c'est sur Victor Noir qu'on frotte dessus ! " ${ }^{11}$; quelques Français : «C'est le porte-bonheur ! Il est beaucoup touché, c'est pas mal comme bénédiction ${ }^{12}$.

Il y a aussi, mais plus rarement, des touristes qui se promènent et trouvent la tombe de Victor Noir par hasard, sans savoir de qui il s'agit. Dans ce cas, on rencontre d'habitude de l'intérêt pour la sculpture lustrée, les gens notent le nom du journaliste pour se renseigner ensuite. Un couple français qui passe près de la tombe du journaliste : la femme le remarque et appelle son mari. Ils s'approchent : d'abord, tous deux frottent assez longtemps les pieds de Victor ; ensuite la femme touche le sexe : "C'est qui ça ?... C'est quoi ça ?... C'est bizarre ! " ${ }^{13}$. On note que le gisant, tel qu'il paraît actuellement, permet non seulement les pratiques d'attouchement mais souvent les provoque en présentant toutes les possibilités d'interprétation. Il faut remarquer qu'assez souvent les actions rituelles s'accomplissent spontanément. Il y a ceux qui viennent vers cette tombe sans savoir exactement ce qu'il faut faire ni comment pour obtenir les biens désirés ou vérifier si cela marche; ils sont alors, sur le plan des rites, conduits par une sorte d'intuition qui leur suggère tel ou tel comportement.

D’une certaine manière, ils trouvent sur place «le matériel didactique » et leur interprétation dépend souvent du but de la visite. Les pratiques rituelles se forment assez logiquement en conformité avec la forme du gisant, sa réputation extraordinaire et la spécificité des buts de la visite. On explique, par exemple, que, pour avoir des enfants, il faut toucher le sexe de la statue, s'allonger sur elle, s'asseoir sur elle (ou sur ses pieds); pour rencontrer l'amour de toute une vie, il faut toucher ses pieds; pour faire revenir la personne qu'on aime, il faut embrasser (souvent par trois fois) les lèvres, le menton, le nez de Victor Noir ou mettre un doigt sur son cœur (l'impact de la balle). Il existe même un rapport entre l'attouchement des pieds de Victor Noir et le nombre des enfants désirés.

10. Observation du 14-05-06.

11. Observation du 17-05-06.

12. Ibid.

13. Observation du 12-07-06. 
Certaines personnes, après quelques attouchements, se mettent devant le gisant, du côté des pieds, et restent ainsi un certain temps comme plongées dans la méditation. Parfois elles s'assoient à côté de la tête et mettent la main sur le cœur. Cela donne l'impression qu'elles essaient d'établir un contact avec Victor Noir en lui transmettant leurs demandes et leurs problèmes. Très souvent, les admirateurs touchent quelques parties, font ensuite cette espèce de méditation et, avant de partir, répètent leurs attouchements. Certains, avant de s'en aller, bénissent la statue d'un signe de croix.

S'il s'agit d'un couple, on constate des variations de rites. Par exemple, une femme touche la statue en demandant de l'aide à Victor Noir tandis que l'homme l'attend sérieusement sur le côté. Dans certains cas, l'homme prend part à certaines pratiques : tous deux touchent le sexe de Victor Noir, ou chacun touche un des pieds de la statue et ils restent ainsi un certain temps. Un couple africain, fréquentant la tombe régulièrement, opère le rite suivant : la femme s'assied sur le gisant pendant que l'homme tient les pieds de Victor Noir. Puis la femme se lève, fait le tour de la statue (à ce moment-là l'homme reste sans rien toucher); ensuite, elle s'assied à nouveau (l'homme, de nouveau, touche les pieds du gisant). Ils répètent ce rite trois fois. Parfois ils y ajoutent l'attouchement de toutes les parties lustrées du gisant et l'homme pousse le sexe du gisant trois fois avec ses mains. Les guides racontent qu'on trouve des préservatifs sur la tombe du journaliste, ce qui donnerait à penser que certains couples font l'amour directement sur le gisant. Parfois, les informateurs livrent des précisions personnelles concernant des rites : "Il faut y croire, sinon ça marche pas " ${ }^{14}$; " Il faut venir beaucoup de fois, quand tu ne travailles pas, quand tu as du temps... » ${ }^{15}$; " Il faut venir le premier dimanche du mois ${ }^{16}$.

Sur la tombe, on trouve toujours des fleurs fraîches qui prouvent, en quelque sorte, l'existence du culte. Les gens apportent des fleurs, soit pour remercier Victor Noir de les avoir exaucés ou d'avoir comblé leurs désirs, soit pour l'amadouer. En réalité, ils le font à titre d'offrande. Les informateurs expliquent qu'il faut déposer les fleurs dans le chapeau, dans la main de Victor Noir ou entre ses jambes. Aux mêmes endroits, les visiteurs déposent des messages avec leurs demandes ${ }^{17}$ : «Donne-moi un bel homme. On t'aime »; "Victor Noir faites que je puisse me marier et avoir des enfants "; "Fais-nous avoir un bébé ", etc.

\footnotetext{
14. Observation du 17-05-06.

15. Observation du 25-05-06.

16. Observation du 18-05-06 (l'informateur est un gardien du cimetière).

17. Sur les messages dans un contexte catholique $c f$. Bonnet, 1976.
} 


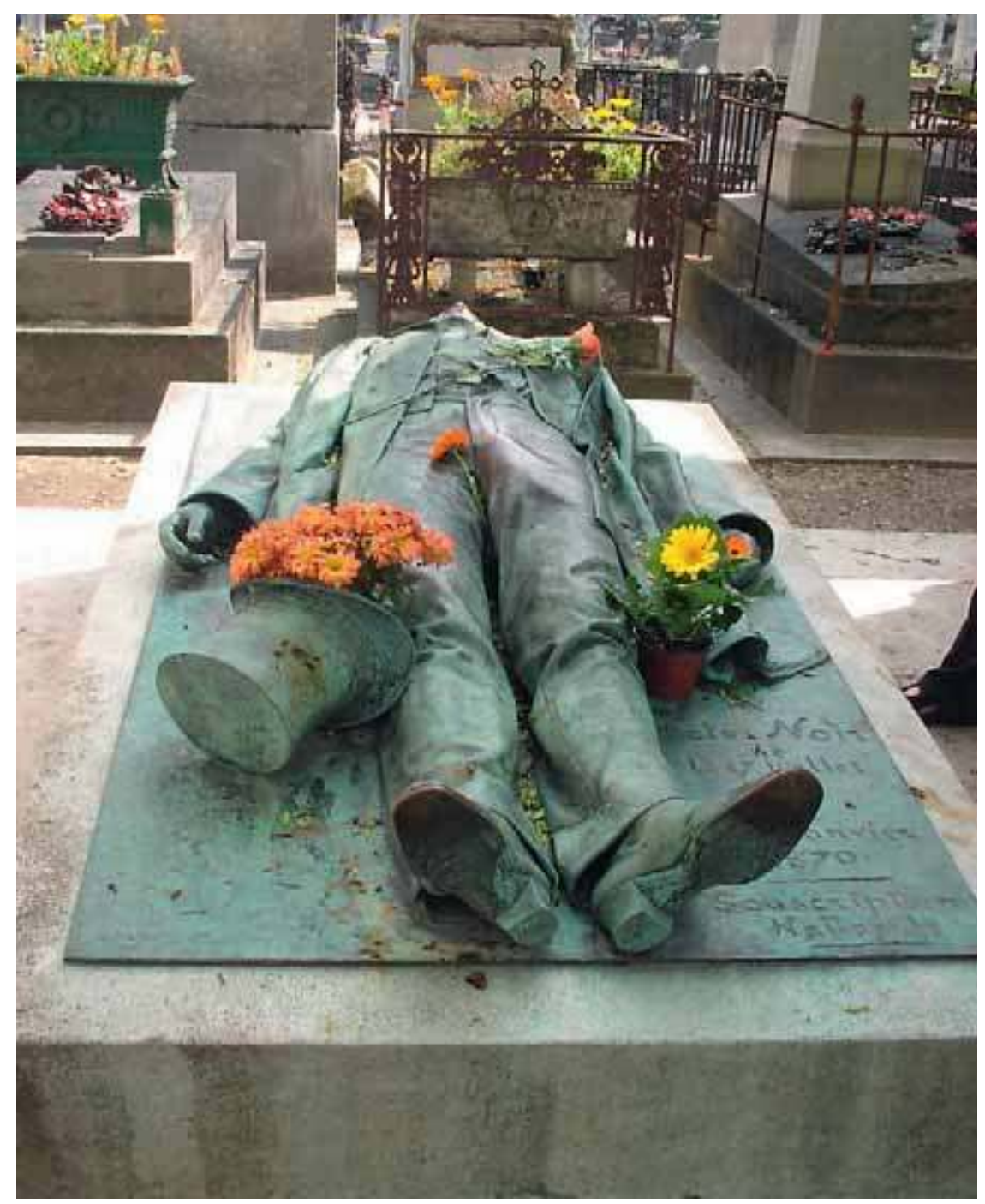

Les fleurs dans le chapeau, sur le sexe ou dans les mains de Victor Noir (photo de l'auteur, mai-juin 2006)

D'après les recherches effectuées, on a pu distinguer le culte politique de Victor Noir, né juste après sa mort, et le culte sexuel, apparu probablement au cours des années soixante. Le premier type de culte est lié au nom du journaliste républicain, martyr et héros de la République ; le second est attaché à la statue d'un beau jeune homme tué à l'âge de vingt-deux ans, deux jours avant son mariage. Ces deux sens du culte coexistent aujourd'hui avec un évident avantage pour le second. 
Il faut souligner particulièrement le fait de la «mutation de la fonction » et de la "mutation du sens » ${ }^{18}$. À la fin du XIX ${ }^{\mathrm{e}}$ siècle, la statue de Victor Noir était considérée dans l'esprit du culte "politique » rendu au journaliste. Tous les détails de la sculpture soulignaient, pour le spectateur de l'époque, la mort brutale d'un journaliste tué froidement par le cousin de l'empereur. Son costume était neuf, destiné à son mariage : c'est pour mieux jouer son rôle de témoin, pour se rendre bien habillé chez un prince que Victor Noir l'avait revêtu. La chemise et le pantalon déboutonnés montrent les dernières minutes de sa vie, quand ses amis voulaient sans succès l'aider à respirer. Tout cela devait faire comprendre au spectateur le tragique de cette mort et le faire frémir devant sa brutalité. Cette vision est réinterprétée au $\mathrm{XX}^{\mathrm{e}}$ siècle. La sculpture est devenue objet de plaisanteries, de photos indécentes, de rites de fécondité. La vie et la mort du journaliste sont réinterprétées sous un angle érotique. Les mêmes détails de la sculpture sont expliqués autrement. On dit qu'il est bien habillé parce qu'il était un dandy, un Don Juan, qu'il avait eu beaucoup de femmes et qu'il avait été tué à cause de l'une d'elles. On peut deviner pourquoi ses vêtements sont déboutonnés. On dit aussi que son sexe "a beaucoup travaillé » pendant sa vie et qu'il était en érection même au moment de sa mort. Ce sont là quelques transformations et mutations qu'a connues le culte entourant la mémoire de Victor Noir.

L'étude du phénomène dans sa dynamique a permis de remarquer que le nombre des adeptes du culte, comme celui des fonctions du gisant, augmente avec le temps et que ces dernières deviennent de plus en plus universelles. Ainsi, dans les années soixante, on associait au gisant exclusivement le pouvoir d'aider les femmes stériles; dans les années soixante-dix, on y ajoute la capacité de ranimer les virilités défaillantes des hommes, d'aider à toute sorte de problèmes d'amour (se marier, faire revenir la personne aimée) ; aujourd'hui, les guides racontent que l'attouchement du gisant à l'endroit du sexe porte bonheur toute l'année. De même, des fonctions du gisant telles que "Faire revenir la personne qu'on aime » et "Aider à toute sorte de problèmes d'amour » se transforment dans la conscience des gens en demandes plus concrètes. Par exemple, une jeune femme est persuadée que Victor l'aide, parce que la première fois qu'elle a fait le vœu d'une rencontre, cela a «marché ».

L'administration du cimetière n'apprécie guère ce qui se passe autour de la tombe de Victor Noir. En 2004, la sépulture a été entourée de barrières. Pascal Daniel, chef du service des cimetières de la ville de Paris, explique : "Cette tombe a été régulièrement l'objet d'attouchements. Des usagers du cimetière se sont plaints après avoir vu des jeunes femmes frotter leur sexe sur celui de Victor Noir, ou sur ses chaussures. Ces gestes sont pénibles et choquants. Il est anormal de devoir entourer une sépulture d'une barrière mais une minorité de personnes

18. Selon les termes du philologue russe J. Tynjanov. 
irrationnelles nous y pousse... Les barrières qui protègent le tombeau de Victor Noir sont provisoires. Mais une protection plus solide n'est pas encore programmée. Nous ne pouvons faire de travaux sans l'accord des ayants droit » (Cloris, 2004). Mais cette protection n'a pas résisté longtemps : au cours d'une manifestation devant la tombe, les barrières ont été enlevées par Yves Contassot, adjoint au maire chargé des Jardins en novembre 2004. La situation mérite d'être racontée.

Selon Le Parisien du 6 novembre, c'était un canular lié à la tombe du pauvre Victor Noir : "Yves Contassot est tombé dans le panneau... Hier matin, il s'est retrouvé face à une dizaine de jeunes femmes munies de banderoles et d'un mégaphone scandant: "Les barrières en arrière! Victor on veut ton corps !" De simples citoyennes frustrées d'être privées de leur idole? C'est en tout cas ce qu'a cru l'élu parisien. Lorsqu' "Isabelle”, une jeune femme visiblement enceinte, prétend avoir été fertilisée par le mort “en se frottant”, Contassot réplique très sérieusement qu'il "n'a rien contre les croyances". Or la meneuse de cette troupe déchaînée n'était autre que Péri Cochin, chroniqueuse dans l'émission “On a tout essayé", animée par Laurent Ruquier sur France 2 ! Yves Contassot, qui a fait retirer hier les barrières de la censure, aurait gagné à passer un simple coup de fil au responsable du cimetière !" (Torgemen, 2004 : 3).

Bien que, dans cet article et dans l'émission elle-même, on explique qu'il s'agissait d'une farce, présentant la tombe de Victor Noir non pas comme un lieu de culte, mais comme un lieu de plaisanterie, dans Le journal de la nuit, sur France 2, cet événement est exposé d'une autre manière. Le journaliste, parmi d'autres nouvelles, raconte très sérieusement comment l'adjoint du maire, responsable des cimetières, Yves Contassot, a rendu la statue de Victor Noir à ses adeptes et comment les barrières ont été retirées par décision de la Mairie de Paris. Le petit reportage de la manifestation montre la femme enceinte qui est sur place pour prouver les pouvoirs magiques du gisant et exiger l'enlèvement des barrières. Le guide-conférencier du cimetière, Marika Vayssair, raconte : "On voit des petits chaussons dans le chapeau. Alors ça doit marcher effectivement ". Ainsi désormais, malgré le mécontentement de l'administration du cimetière, le culte prospère : l'histoire de la manifestation a contribué à sa popularisation...

Il est important de remarquer également le rôle des livres (guides, histoire populaire) et des médias (la presse, Internet) dans la popularisation du culte lié à la tombe de Victor Noir. Les auteurs de nombreux guides ou articles de presse ont joué un rôle décisif dans la formation des représentations liées à la tombe et ont contribué à sa popularité. Internet a joué un rôle particulièrement important car il a permis une création illimitée et incontrôlée d'informations et leur large circulation. Le développement du tourisme de masse, l'accessibilité de l'information ont contribué à la popularisation de ce culte et à la diffusion de l'information le concernant. Cela attirait et attire encore le public. 
Il aurait été également nécessaire, bien que très hasardeux car difficile à étayer, de repérer l'influence de l'évolution socioculturelle globale sur l'apparition et l'évolution du culte de la fécondité. On peut émettre l'hypothèse que la révolution sexuelle vécue par le monde occidental dans les années soixante et soixante-dix a joué un grand rôle. Il est probable que les évolutions de la conscience collective sous l'influence de cette révolution ont fourni d'importants éléments et les conditions favorables à l'épanouissement d'un nouveau culte. Il est, enfin, vraisemblable que, si l'hypothèse du canular est la bonne, les étudiants qui l'ont monté se situaient dans la tradition du canular estudiantin, souvent marqué sexuellement. En outre, il faut évoquer le renforcement, dans la société française des années soixante, de l'intérêt pour l'occultisme, la magie, la mystique, pour tout ce qui a trait à l'au-delà. Ce n'est pas donc par hasard que, parmi les admirateurs de la tombe de Victor Noir, il y ait beaucoup de spirites et de médiums : " Les fakirs, voyants, guérisseurs, thaumaturges, astrologues (Le ministère de l'Intérieur a même son astrologue) dont le rôle considérable et trop peu connu s'étend dans toutes les couches de la société industrielle, nous révèle l'importance de ce folklore urbain. Une baraque foraine sur six recèle en son creux tapissé la prédiction magique de l'avenir. Soixante mille Français spirites communiquent chaque nuit avec les esprits, mais des millions ont peur des ténèbres, perdus dans la forêt ou seuls dans un lieu maudit; des millions frémissent au théâtre ou au cinéma à l'apparition du spectre, des millions possèdent fétiches et porte-bonheur, prennent garde aux nombres fatidiques, évitent les échelles, consultent l'horoscope... Tout ceci nous montre que le contenu préhistorique est plus qu'un savoir conservé, c'est un feu qu'entretiennent vitalement les déséquilibres et les désadaptations des sociétés évoluées... Le spiritisme est la renaissance des croyances les plus archaïques concernant le double » (Morin, 1970 : 157-160).

Pour expliquer le phénomène, on a dégagé, entre autres par analogie, plusieurs niveaux d'explication. Le premier peut être nommé par convention « historique », car il est lié à la vénération traditionnelle des objets ou lieux sacrés. Il existe des liens qui, traditionnellement, rapprochent la mort et la fécondité dans le folklore de certains peuples. De plus, les pratiques liées au gisant de Victor Noir ressemblent à celles qui se déroulent près de statues de saints, de pierres cultuelles, de tombes vénérées dans la «culture populaire ». Ainsi, en comparant les actions rituelles autour du gisant de Victor Noir avec celles qui sont décrites dans des ouvrages consacrés aux cultes dans la culture populaire, on peut constater qu'elles présentent parfois des analogies directes. Cependant, il semble indispensable d'observer, à côté de ces ressemblances, une différence majeure. C'est que d'habitude, dans la «culture populaire ", l'objet thaumaturgique ne peut pas être objet de plaisanteries ou de dérision. Il existe même une série de récits racontant des cas de châtiments subis par des personnes qui ont traité l'objet de la dévotion sans le respect qui lui est dû. 
En outre, quelque chose rapproche les représentations liées au culte de Victor Noir et le culte des saints tant officiels que populaires, morts de mort violente. Comme l'écrit Jean-Claude Schmitt, «le texte hagiographique dans la culture populaire est d'abord un langage du corps, du fidèle comme de celui du saint : d'où le thème privilégie de la mort violente du saint, d'où le succès des martyrs, dont le sang écoulé, en même temps qu'il manifeste les souffrances du saint, garantit son pouvoir de guérir les souffrances des hommes » (1983 : 19). M.-C. Pouchelle a relevé de nombreux parallèles entre le culte rendu par ses fans à Claude François et le culte des saints (1983). Un thème qui n'est pas absent du culte de Victor Noir après sa mort tragique.

Le deuxième niveau d'explication est lié aux cultes "touristiques » : "Le tourisme a longtemps été perçu comme un facteur de dépossession du patrimoine culturel et de falsification des traditions. Associé à la superficialité, au simulacre, à la duperie, il crée un terrain propice à l'invention des traditions "inauthentiques » (Le Menestrel, 2004 : 199). « Si le tourisme est une religion, l'infrastructure touristique est une vaste Église... », écrit Jean-Didier Urbain (2002 : 306). En poursuivant l'analogie, on peut parler des "lieux sacrés " du tourisme qui sont propres à chaque ville et auxquels sont liés des " rites " spécifiques : ce sont ainsi les plus beaux points de vue panoramiques sur la ville où les visiteurs laissent des graffitis; des "arbres des désirs" sur lesquels il faut nouer des rubans en formulant des vœux; des fontaines dans lesquelles il faut jeter des pièces de monnaie pour revenir à cet endroit ou pour avoir de la chance; diverses statues qu'il faut frotter pour obtenir le bonheur ou favoriser sa carrière, etc. Les monuments devenus objets d'attouchement existent dans le monde entier : on peut citer de nombreux exemples de statues frottées en raison de leurs pouvoirs magiques dans des villes modernes : des monuments sur le pont Charles à Prague; la statue d'Everard t'Serclaes sur la Grand-Place à Bruxelles; des lions de bronze près de la Résidence à Munich dont il faut toucher le mufle ; un chien de bronze dans une station du métro à Moscou dont il faut toucher le museau en demandant de l'aide pour les événements importants de la journée; un âne dans le groupe de monuments devant Conventa Ceta à Riga ; un sanglier sur le marché central à Florence à qui il faut toucher le groin et donner une pièce de monnaie, etc. Ce sont des phénomènes très répandus mais assez récents et c'est d'eux que le culte de la tombe de Victor Noir se rapproche. Il y a toujours beaucoup de personnes à côté de ces statues, les guides y amènent des touristes, les habitants de la ville les montrent à leurs amis. On y retrouve les même catégories de public que celles repérées au Père-Lachaise : des gens qui croient et viennent assez régulièrement ou ceux qui touchent les statues « au cas où », en se disant "pourquoi pas »; certains qui le font pour s'amuser ou pour prendre des photos; d'autres qui les regardent ou les montrent.

Le troisième niveau est psychologique et lié à l'atmosphère du cimetière qui prédispose à l'apparition des légendes, des rites, des cultes. On a déjà dit que le 
cimetière du Père-Lachaise est un endroit très particulier, où " on se sent envahi d'une sorte d'ambiance mélancolique, compréhensive, qui donne l'illusion que de grands secrets vont se résoudre devant vous. C'est le lieu de méditation par excellence, un site particulier et, dans sa tradition musicale, un chant grégorien. Les noms inscrits sur les tombes, avec les dates, prennent une signification profonde, une résonance infinie et douce... Autour des tombes, des silhouettes passent, portant un arrosoir plein d'eau, une époussette, des brassées de fleurs. La tendresse désolée fait le ménage des morts. On se dit aussi que dans une goutte de temps, deux, trois cents ans, rien ne subsistera plus de ses reliefs, de nos reliefs, et que les grands arbres auront encore grandi. Déjà les sépultures de 1820 sont entrées dans la terre, dans le néant, désagrégées, comme un souvenir de peu d'importance dans la mémoire... Une promenade dans les quartiers anciens de cette nécropole, où les arbres, les bosquets en font encore un jardin suspendu sur la ville, nous ménage un enseignement particulier : dans le dédale de sépultures, le végétal étreint parfois la pierre, la malmenant, la renversant, nous signifiant qu'au-delà du dur désir de durer inscrit dans les pierres tumulaires, dans les statues, la vie, l'arbre jaillit dans son éternel recommencement, faisant de ce paysage de pierres et d'arbres une allégorie du jardin antique, "paysage sacré" célébrant la communion de la vie et de la mort» (Racine, 1997 : 209-210).

André Chabot remarque le climat érotique des cimetières : "Endormi ou mieux encore mort, l'image de pierre, de bronze, l'être aimé inanimé, incapable de voir, d'entendre, de juger, libère les impulsions les plus secrètes, autorise les vertiges les plus inavouables, face à l'inquiétante étrangeté, l'Unheimlich suprême : douter qu'un objet sans vie soit animé. Climat érotique sans rapport avec la santé épanouie, dans lequel l'obsession, le deuil, la mélancolie, ou plus simplement le manque d'être élaborent des fantasmes au milieu de l'ombre et du mystère, de l'apparat funèbre et des apparences équivoques de la sculpture funéraire. Insondables voies de la révélation érotique qui suit les lignes de la matière, vivantes pourvu que l'imagination leur prête vie. Frontières incertaines entre le réel et l'imaginaire. Épiphanie née des hasards, des rencontres et du désir permanent de modeler le monde au gré de ses caprices, dans l'attente toujours en éveil de l'exaltant et du merveilleux... le cimetière, derrière ses murs de quartier réservé, apparaît, pour qui ne pénètre pas en service commandé, comme l'un des rares et derniers refuges du rêve et de l'illusion, du différent et de l'inconnu, de l'extraordinaire et du magique... L'érotisme affectionne le silence, le secret, l'intimité des lieux écartés et le cimetière lui propose précisément un ailleurs, presque un autre monde, presque l'“autre monde” "(1989: 10-11).

Toutes ces explications ne sont pas exhaustives mais on est porté à croire que l'apparition dans la vie quotidienne et la culture des sociétés modernes de traditions nouvelles par leur contenu, mais archaïques par leur forme, est un trait de notre temps. Effectivement, on a à faire à une mode portant vers des " mythologies » récentes créées autour d'une ville, des manifestations diverses 
du folklore urbain. Il semble que chaque ville aspire à posséder son endroit exotique où il faut toucher quelque chose, laisser quelque chose, écrire quelque chose pour obtenir des grâces diverses. Cela peut être une pierre, un arbre, une statue, une tombe, etc. Cet objet peut avoir son histoire qui correspond au culte, qui n'est pas lié avec lui, ou cela peut être tout simplement un culte inventé. Ces objets s'entourent de légendes et de rites, ils sont recherchés par les touristes qui, en voyageant à travers le monde, sont habitués à les rencontrer. Les guides deviennent porteurs et propagateurs de l'information et provoquent le développement de cultes semblables.

"Le concept de "tradition inventée" commença à intéresser quelques historiens dans les années 1970. Ils étaient frappés par le fait que certaines nouvelles pratiques sociales, ritualisées ou exprimées en symboles... revendiquaient une continuité avec le passé... Ces innovations cachées derrière l'apparence de l'ancienneté n'étaient pas confinées dans ce que la théorie de la modernisation appelle les “sociétés traditionnelles" (Dimitrijevic, 2004 : 7). Le culte sexuel autour de la tombe de Victor Noir peut être présenté sous la forme d'un processus d'invention de la tradition car il s'agit d'un culte dont les adeptes revendiquent la légitimité en recréant des liens imaginaires avec le passé.

Marina EMELYANOVA-GRIVA

EHESS - Centre d'anthropologie religieuse européenne marina.emelyanova@gmail.com

\section{Bibliographie}

BerTRAND Régis, Vovelle Michel, 1983, La Ville des morts. Essai sur l'imaginaire urbain contemporain d'après les cimetières provençaux, Paris, Éditions du Centre national de la recherche scientifique.

Bonnet Serge, 1976, Prières secrètes des Français d'aujourd'hui, Paris, Éditions du Cerf. Снавот André, 1989, Érotique du cimetière, Paris, Henry Veyrier.

Charneau Roger, 1997, Les Ailes et le Sablier, Paris, Le Cercle d'Art.

Cloris J., 2004, "Une sépulture protégée des "frottements indécents ", Le Parisien, 7 novembre 2004.

Dimitrijevic Dejan, (éd.), 2004, Fabrication des traditions, invention de modernité, Paris, Éditions de la Maison des Sciences de l'Homme.

Hobsbawm Eric, Ranger Terence, (eds.), 1983, The Invention of Tradition, Cambridge, Cambridge University Press.

LaPLANTINe François, 2002, La description ethnographique, Paris, Nathan.

LAPLANTINE Gérard, 1993, "Inscriptions lapidaires et traces de passages : formation de langages et de rites ", in Ethnologie des faits religieux en Europe, Paris, CTHS, pp. 137-159.

LASSÈRE Madeleine, 1997, Villes et cimetières en France de l'Ancien régime à nos jours: le territoire des morts, Paris, L'Harmattan. 
Le Menestrel Sara, 2004, " Du bon usage des "bons temps" : la mise en scène touristique des festivals cadiens en Louisiane ", in Dimitrijevic D., (éd.), Fabrication des traditions, l'invention de modernité, Paris, Éditions de la Maison des Sciences de l'Homme, pp. 199-210.

Le Normand-Romain Antoinette, 1995, Mémoire de marbre, Paris, Mairie de Paris.

Lloyd Jill, 2002, "Christian Schad, réalité et illusion ", in Christian Schad, Peintures, dessins, schadographies, Munich, Schirmer/Mosel, pp. 13-26.

LOURIE V., 2003, « Pamjatnik v gorode : ritual'no-mifologicheskij kontekst » [Le monument dans la ville : les contextes rituelles et mythologiques], in Sovremennyj gorodskoj fol'klor [Le folklore urbain contemporain], Moskva, RGGU, pp. 420-429.

Morin Edgar, 1970, L'homme et la mort, Paris, Seuil.

PouChElle Marie-Claude, 1983, «Sentiment religieux et show business : Claude François objet de dévotion populaire ", in Schmitt J.-C., Les saints et les stars, Paris, Beauchesne, pp. 277-299.

Racine Michel, 1997, Jardins en France, Paris, Actes Sud.

SCHміт Jean-Claude, 1997, Les saints et les stars : le texte hagiographique dans la culture populaire, Paris, Beauchesne.

TARTAKovsky Danielle, 1999, Nous irons chanter sur vos tombes: le Père-Lachaise, XIXe$\mathrm{XX}$ siècle, Paris, Aubier.

Togemen E., 2004, "Contassot piégé par Ruquier devant la tombe de Victor Noir ", Le Parisien, 6 novembre 2004.

Urbain Jean-Didier, 2002, L'idiot du voyage, Paris, Payot.

VAn Gennep Arnold, 1982, Manuel de folklore français contemporain, 1, Paris, A. et J. Picard.

XXX, 1990, "Le culte des grands hommes : du cimetière à la galerie ", L'art du $19^{e}$ siècle, Paris, Editio, pp. 212-214.

ZÉvAÈs Alexandre, 1929, L'affaire Pierre Bonaparte : le meurtre de Victor Noir, Paris, Librairie Hachette.

\section{Résumé}

La tombe du journaliste Yvan Salmon, dit Victor Noir (1848-1870), est aujourd'bui l'un des monuments les plus visités du cimetière parisien du Père-Lachaise; elle fait même l'objet d'un véritable culte. La légende veut qu'en frottant le gisant, surtout à l'endroit du sexe, on recouvre fécondité ou virilité. L'étude s'attache à montrer quelles transformations fonctionnelles a connu le gisant de Victor Noir depuis son inauguration jusqu'à nos jours. Le culte originel est politique: journaliste d'opposition, Victor Noir est tué le 10 janvier 1870 lors d'une altercation avec le prince Pierre Bonaparte, cousin de Napoléon III ; ses obsèques réunissent plus de cent mille personnes; il devient le symbole de la répression de l'Empire face à la lutte pour la liberté. Le sculpteur républicain Jules Dalou édifie à sa mémoire, en 1891, un gisant de bronze. Dans le courant $d u \mathrm{XX}^{e}$ siècle, sa tombe acquiert progressivement des connotations et des vertus érotiques: un culte sexuel se substitue définitivement au culte politique à partir des années soixante.

Mots-clés : cimetière, culte, fécondité, folklore urbain, gisant. 
IO8 - ARCHIVES DE SCIENCES SOCIALES DES RELIGIONS

\section{Abstract}

The tomb of Yvan Salmon (alias Victor Noir) is among the most frequently visited monuments in the Parisian Père-Lachaise cemetery monuments. It has become an object of worship. According to common belief, rubbing Noir's recumbent effigy, especially his sexual organ, brings fertility and masculine vigor. The present study aims at revealing the functional transformations of Victor Noir's monument from its creation, to the present. Originally, the cult related to the monument was political: Victor Noir, a journalist critical of the Imperial regime, was killed on January 10th, 1870 by Prince Pierre Bonaparte (Emperor Napoleon III's cousin). Over one hundred thousand people attended his funeral. He became a symbol of imperial repression and the struggle for freedom. In 1891, the Republican sculptor Jules Dalou made a magnificent recumbent bronze effigy of the hero. In the mid-twentieth century, the tomb was progressively attributed with hidden and erotic powers. During the 1960s, sexual worship definitively replaced the political one.

Key words: cemetery, cult, fecundity, urban folklore, recumbent statue.

\section{Resumen}

La tumba del periodista Yvan Salmon, conocido como Victor Noir (1848-1870) es hoy uno de los monumentos más visitados del cementerio parisino de Père-Lachaise, $y$ es objeto de un verdadero culto. La leyenda cuenta que frotando la estatua yacente, sobre todo en el lugar del sexo, se obtienen fecundidad o virilidad. El estudio se propone mostrar qué transformaciones funcionales ha conocido la estatua de Victor Noir desde su inauguración hasta nuestros días. El culto original es político: periodista de oposición, Victor Noir es asesinado el 10 de enero de 1870 durante un altercado con el príncipe Pierre Bonaparte, primo de Napoleón III. Sus funerales reúnen más de cien mil personas: se vuelve así un símbolo de la represión del Imperio frente a la lucha por la libertad. El escultor republicano Jules Dalou realiza en su memoria, en 1891, una estatua yacente de bronce. Durante el siglo XX, su tumba adquiere progresivamente connotaciones y virtudes eróticas: el culto sexual se sustituye definitivamente al culto político a partir de los años sesenta.

Palabras clave: cementerio, culto, fecundidad, folklore urbano, estatua yacente. 